\title{
1 An interventional Soylent diet increases the 2 Bacteroidetes to Firmicutes ratio in human gut 3 microbiome communities: a randomized controlled 4 trial
}

5 Ryan H. Hsu ${ }^{1 *}$, Dylan M. McCormick ${ }^{2}$, Mitchell J. Seitz Jr. ${ }^{3}$, Lauren M. Lui ${ }^{4}$, Harneet S. 6 Rishi $^{5,6}$, Adam P. Arkin ${ }^{4,7}$

8 'Plant and Microbial Biology, University of California, Berkeley, California, United States 9 of America

${ }^{2}$ Molecular and Cell Biology, University of California, Berkeley, California, United States 12 of America

${ }^{3}$ Integrative Biology, University of California, Berkeley, California, United States of 15 America

${ }^{4}$ Environmental Genomics and Systems Biology, Lawrence Berkeley National Laboratory, Berkeley, California, United States of America

${ }^{5}$ Biophysics Graduate Group, University of California, Berkeley, California, United States of America

${ }^{6}$ Designated Emphasis Program in Computational and Genomic Biology, University of California, Berkeley, California, United States of America

30 E-mail: ryan.hsu@berkeley.edu $(\mathrm{RH})$ 


\section{Abstract}

33 Our knowledge of the relationship between the gut microbiome and health has rapidly

34 expanded in recent years. Diet has been shown to have causative effects on

35 microbiome composition, which can have subsequent implications on health. Soylent

362.0 is a liquid meal replacement drink that satisfies nearly $20 \%$ of all recommended

37 daily intakes per serving. This study aims to characterize the changes in gut microbiota

38 composition resulting from a short-term Soylent diet. Fourteen participants were

39 separated into two groups: 5 in the regular diet group and 9 in the Soylent diet group.

40 The regular diet group maintained a diet closely resembling self-reported regular diets.

41 The Soylent diet group underwent three dietary phases: A) a regular diet for 2 days, B)

42 a Soylent-only diet (five servings of Soylent daily and water as needed) for 4 days, and

43 C) a regular diet for 4 days. Daily logs self-reporting diet, Bristol stool ratings, and any

44 abdominal discomfort were electronically submitted. Eight fecal samples per participant

45 were collected using fecal sampling kits, which were subsequently sent to uBiome, Inc.

46 for sample processing and V4 16S rDNA sequencing. Reads were clustered into

47 operational taxonomic units (OTUs) and taxonomically identified against the

48 GreenGenes 16S database. We find that an individual's alpha-diversity is not

49 significantly altered during a Soylent-only diet. In addition, principal coordinate analysis

50 using the unweighted UniFrac distance metric shows samples cluster strongly by

51 individual and not by dietary phase. Among Soylent dieters, we find a significant

52 increase in the ratio of Bacteroidetes to Firmicutes abundance, which is associated with 
53 several positive health outcomes, including reduced risks of obesity and intestinal

54 inflammation.

\section{Introduction}

56 The gut microbiota plays a key role in mediating human health, including aspects of

57 infection, inflammation, and obesity [1-3]. While many factors that influence the gut

58 microbiome have been identified, diet has been consistently shown to be a major

59 contributor $[4,5]$. This has led to a growing consumer awareness of dietary choices that

60 function as prebiotics and probiotics [6].

61 Meal replacement drinks are an emerging alternative to traditionally prepared meals that

62 are designed to conveniently supply full servings of dietary nutrients. Among these

63 products, Soylent 2.0 in particular has gained recent popularity. Soylent 2.0's

64 formulation is engineered to fulfill nearly all recommended daily intakes of total fat,

65 sodium, potassium, carbohydrates, proteins, vitamins, and minerals, without excess

66 sugars or cholesterol [7]. Given the critical role of intestinal flora in various human

67 health outcomes, we are interested in studying how meal replacements like Soylent

68 affect gut microbiome composition.

69 To assess the compositional changes to the gut microbiome resulting from a Soylent-

70 only diet, we performed a parallel randomized controlled trial consisting of a control

71 group that adhered to self-reported regular diets and a treatment group that received a

72 Soylent 2.0 dietary intervention. Fecal samples were regularly collected and sequenced

73 to quantify microbial populations at defined timepoints throughout the study. 
74 This study was conceived, designed, and coordinated by a team of undergraduates at

75 UC Berkeley and funded via Experiment, a website that hosts online crowdfunding

76 campaigns for scientific research. The funders did not participate in study conception,

77 experimental design, or data analysis.

\section{Material and Methods}

\section{Institutional clinical trial registration}

80 The study was granted institutional review board approval from the Committee for

81 Protection of Human Subjects at the University of California, Berkeley (CPHS \#2016-04-

828727 , October 14, 2016). The trial was publicly submitted to the clinicaltrials.gov registry

83 (ID \#NCT03203044, June 27, 2017) following completion. The trial was not publicly

84 registered prior to subject enrollment due to a miscategorization of the study as a non-

85 clinical trial. All related and future trials will be prospectively submitted to a public 86 registry.

\section{Subject enrollment and selection}

88 Written informed consent to participate in the study was solicited through Mycrobes, an

89 undergraduate student organization at the University of California, Berkeley. Consenting

90 individuals were administered a questionnaire surveying for age, biological sex,

91 ethnicity, student status, pregnancy status, food allergies or sensitivities, health

92 complications, dietary supplements or medications, prior Soylent consumption, and 
93 dietary and lifestyle descriptions. Participants were selected at random from eligible

94 individuals who did not report non-student status, pregnancy, serious food allergies or

95 sensitivities, chronic disease, current use of medications, or prior Soylent consumption

96 (Fig 1). Participation in the study was entirely voluntary, and subjects did not receive

97 any compensation.

98 Fig 1. CONSORT flow diagram of the study.

99 Study procedure

100 The study design is a parallel randomized controlled trial. Participants were randomly

101 assigned to the control or treatment group, also referred to as the regular diet and

102 Soylent diet group. Individuals in the control group maintained their regular diets

103 throughout the study, allowing characterization of daily fluctuations in microbiome

104 composition, while participants in the treatment group received 20 bottles of Soylent 2.0

105 to consume during phase B of the study. Soylent 2.0 is a liquid formulation consisting of 106 primarily soy protein, algal oil, and isomaltulose, as well as smaller amounts of other

107 ingredients such as essential vitamins and minerals (S1 Fig).

108 The study was organized into three phases spanning a period of ten days (Fig 2).

109 During phase A, all participants maintained their regular diets for two days. In phase B,

110 the Soylent diet group switched to a Soylent-only diet consisting of a recommended 5

111 servings of Soylent daily ad libitum (and water as needed) for four days, while the

112 regular diet group retained a normal diet. During Phase C, all participants returned to

113 their regular diets for four days. Prior to the initiation of the study, eight uBiome stool 
114 sampling kits were distributed to each participant for use on eight specific days.

115 Participants who missed a sampling day were instructed to sample on the following day

116 if possible. Additionally, participants submitted daily electronic forms reporting their diet,

117 time of bowel movements, Bristol stool ratings, and any symptoms or discomfort (S1

118 File). The primary outcome measure is microbiome composition.

119 Fig 2. Study design. The study design consists of a time-course over 10 days. 9 and 5

120 participants were randomized to the Soylent and regular diet groups, respectively. The

121 Soylent diet group maintained a regular diet during phase $A$ to quantify baseline

122 microbiome composition, switched to a Soylent-only diet during phase $\mathrm{B}$, and returned

123 to a regular diet during phase $\mathrm{C}$. The regular diet group, which serves a control,

124 maintained a regular diet throughout the study period to quantify day-to-day variations in

125 microbiome composition. Fecal samples were collected on the 8 days specified using

126 the uBiome Gut Kit and submitted for $16 \mathrm{~S}$ sequencing.

\section{Sample collection and 16S rDNA sequencing}

128 Commercially available uBiome Gut Kits were used to sample, store, and transport fecal

129 samples to uBiome, Inc [8]. Fecal samples were swabbed from used toilet paper with

130 the included sterile swabs and immediately resuspended into tubes containing lysis and

131 stabilization buffer. The tubes were sealed and stored at ambient temperature. Once all

132 sampling was completed, samples were delivered to uBiome Inc. and run through their

133 standard stool 16S sample processing and sequencing pipeline as follows: Genomic

134 DNA was extracted in a class 1000 clean room using a column-based approach by a 
135 liquid-handling robot. The V4 region of the 16S rRNA gene was amplified using

136 universal primers (515F:GTGCCAGCMGCCGCGGTAA and 806R:105

137 GGACTACHVGGGTWTCTAAT) and barcoded to allow for multiplexed sequencing.

138 Column-based cleanup, size selection, and qPCR quantification were utilized to prepare

139 libraries. 2 x 150 bp paired-end sequencing was performed on an Illumina NextSeq 500.

140 Sequencing reads were demultiplexed using the Illumina BCL2FASTQ algorithm and

141 electronically sent to the researchers.

\section{Read processing and OTU analysis}

143 Custom scripts were used to merge sequencing data across sequencing lanes and

144 relabel samples [9]. USEARCH 9.2 was utilized to stitch and quality filter the reads.

145 Chimeras were identified de-novo and removed with the UCHIME algorithm [10,11].

146 Reads were then clustered and assigned to OTUs at a 0.97 identity threshold using

147 VSEARCH 2.3.4 [12]. Mothur 1.39 .5 was run to assign taxonomy according to the

148 GreenGenes_13_8 16S database, allowing the determination of abundance at near-

149 species resolution in each sample [13,14]. PyNAST 1.2.2 and FastTree were employed

150 to align OTUs and build a tree used to infer phylogenetic distance $[15,16]$. Shannon-

151 Wiener and Gini-Simpson ecological diversity indices and UniFrac distance metrics

152 were calculated using QIIME 1.9.1 [17]. To reduce biases arising from varying

153 sequencing depth, samples with less than 2,000 reads were discarded $(n=6)$, and the

154 remaining samples $(n=62)$ were normalized by rarefying to 2,000 sequences [18].

155 Rarefaction curves and jackknife estimates were generated to validate the 2,000 read

156 threshold (S2 File) [17]. Samples were binned into one of six groups representing the 
157 diet arm (Regular or Soylent) and study phase (A, B, or C). Since it has been shown

158 that stomach contents take about a day to fully reach and pass the intestinal tract,

159 samples are categorized by the dietary phase 24 hours prior to collection [6].

\section{Taxonomic analysis}

161 Intra-sample diversity (a-diversity) was measured using the Shannon-Wiener $(H)$ and

162 Gini-Simpson (D) diversity indices [17]. Calculated a-diversity metrics were averaged

163 within one of six bins representing diet-group and phase of study (e.g. Soylent A). For

164 each individual, the relative abundances of four dominant phyla (Actinobacteria,

165 Bacteroidetes, Firmicutes, and Proteobacteria) were normalized by subtracting the

166 baseline community as established in phase $A$. These values were then binned and

167 averaged in the same manner as the aforementioned a-diversity metrics.

168 Distributions within bins containing at least eight samples (Soylent A, Soylent B,

169 Regular A, and Regular B) were tested for normality using the D'Agostino-Pearson

170 omnibus test. For a-diversity metrics, the Regular A, Soylent A, and Soylent B bins were

171 accepted to follow normal distributions $(p>0.05)$, while the Regular B bin was not $(p<$

172 0.05). For changes in the relative abundance of Bacteroidetes, Firmicutes, and

173 Proteobacteria, the Regular A, Regular B, Soylent A, and Soylent B bins were accepted

174 to follow normal distributions $(p>0.05)$. For changes in the relative abundance of

175 Actinobacteria, the Soylent A and Soylent B bins were accepted to follow normal

176 distributions $(p>0.05)$, while the Regular A and Regular B bins were not $(p<0.05)$. 
177 Differences between these bins were statistically analyzed using two-tailed t-tests, and

$178 p$-values less than 0.05 were considered to be statistically significant.

179

180 Results

181 Selected cohort

182 Study recruitment and follow-ups began on October 20, 2016 and ended on December

183 2, 2016. Informed Consent was received from 29 individuals, and screening forms were

184 subsequently collected to determine which individuals were eligible for participation.

185 Fourteen participants were randomized into either the regular diet or Soylent diet group

186 with a 5:9 allocation ratio using the 'random.permutation' function of the NumPy

187 package for Python 2.7 (Fig 1). The sample size was determined to maximize use of

188 study funding.

189 The 14 participants were all active University of California, Berkeley undergraduates

190 aged 18-21. The regular diet group consisted of 2 females and 3 males, while the

191 Soylent diet group consisted of 3 females and 6 males. Participants were of Asian,

192 European, Hispanic, and Native American descent. Three participants identified as

193 vegetarians, while the rest consumed vegetables and meat on a regular basis (Table 1).

194 Table 1. Baseline sociodemographic and dietary profiles of the participants. 


\section{Quality of collected samples}

196 One individual in the Soylent diet group consumed less Soylent than instructed, and

197 therefore their data was not included in the analysis. Eight of the remaining 104 fecal

198 samples were not collected due to a lack of bowel movements. Of the submitted

199 samples, $71.9 \%$ returned sequencing data from uBiome Inc., with the 1st, 2 nd, and 3rd

200 quartiles of sequencing depths at 8335,83777 , and 208145 reads, respectively (S1

201 Table, S2 Fig). Although we also collected Bristol stool ratings from each participant, the

202 data were inconsistent and did not warrant formal analysis.

$203 \alpha$ - and $\beta$-diversity metrics remain stable during a Soylent diet

204 Shannon-Wiener and Gini-Simpson a-diversity indices did not significantly change from

205 a Soylent dietary intervention (phase A to B) (S3 Fig).

206 Inter-sample diversity ( $\beta$-diversity) was quantified using unweighted and weighted

207 UniFrac, which scores distance between samples according to phylogenetic similarity,

208 in this case using the 16S V4 sequence. $\beta$-diversity was visualized using principal-

209 coordinate analysis (PCoA). Unweighted Unifrac, which only considers the presence or

210 absence of each OTU, shows that samples strongly cluster by individual. No clustering

211 of Soylent-treated samples (those taken from participants in the Soylent diet group

212 during phase B) is observed (Fig 3). Similar clustering patterns are visible using

213 weighted UniFrac, which scores similarity of samples based on OTU abundance as well

214 (S4 Fig). 
215 Fig 3. Principle coordinate analysis and visualization of the unweighted UniFrac

216 metric. A) Unweighted UniFrac, which considers the binary presence or absence of

217 each OTU, reveals that samples cluster strongly by participant (indicated by color) and

218 not by diet (denoted by 's or x's). B) Agglomerative clustering performed on the first ten

219 principal coordinates validates these clustering patterns.

220 Soylent consumption alters relative abundance of dominant

221 phyla

222 During the Soylent dietary intervention (phase B), the Soylent diet group exhibited a 223 significant increase in the abundance of Bacteroidetes $(p=0.011)$, as well as a 224 statistically insignificant decrease in the abundance of Firmicutes $(p=0.078)$, compared

225 to the regular diet group (Fig 4A). Accordingly, the Bacteroidetes to Firmicutes ratio 226 increased significantly during the same time period $(p=0.028)$ (Fig 4B). No significant 227 change in Proteobacteria abundance was observed (Fig 4A). Since the Actinobacteria 228 bins did not follow normal distributions, no comparison was made for the phylum.

229 Fig 4. Abundance changes of dominant gut microbiota phyla. A) Changes in the 230 normalized relative abundance of four dominant gut microbiota phyla averaged within 231 each diet-phase group. In comparison with the regular diet group, the Soylent diet group 232 shows a significant increase in Bacteriodetes $(p=0.011)$ and an insignificant decrease in 233 Firmicutes $(p=0.078)$ abundances during the Soylent dietary intervention (phase B). 234 There is no significant change in Proteobacteria abundance during this phase 235 ( $p=0.937)$. Statistical comparison of Actinobacteria abundance was not performed 
236 because the data was not normally distributed. B) Changes in the Bacteriodetes to

237 Firmicutes ratio. During the Soylent diet intervention (phase B), there is a significant

238 increase in the Bacteroidetes to Firmicutes ratio $(\mathrm{p}=0.028)$. Standard error is shown.

\section{Discussion}

240 Based on calculated Shannon-Wiener and Gini-Simpson diversity scores, we find no

241 significant change in a-diversity across either diet arm, which suggests that overall

242 microbiome diversity is resilient to dietary changes in the short term. Since Soylent is

243 very nutrient-rich, it likely does not starve a large enough fraction of gut microbiota to

244 significantly reduce a-diversity. This finding could also be by the ability of gut microbes

245 to remain dormant during the Soylent dietary intervention. Microbiome diversity is of

246 clinical significance and is negatively associated with diseases such as recurrent

247 Clostridium difficile infection [19]. We find that the interventional Soylent diet does not

248 negatively impact gut microbiota diversity.

249 Unweighted UniFrac reveals that samples cluster strongly within an individual.

250 Furthermore, samples from Soylent diet days among different participants do not cluster

251 together, but rather cluster strongly with each individual's other samples. Visualization

252 of weighted UniFrac, which scores sample similarity based on OTU abundance as well,

253 exhibits similar clustering patterns. The clustering patterns described are less apparent,

254 possibly due to the steep rarefaction that was performed to normalize sample read

255 depth. This suggests that Soylent does not significantly change which organisms are 
256 present in the microbiome, which can be partially explained by the fact that Soylent is

257 pasteurized and therefore cannot act as a probiotic.

258 We find that Soylent consumption significantly increases the Bacteroidetes to Firmicutes

259 ratio in the gut microbiota. Since the Bacteroidetes and Firmicutes together compose

260 over $90 \%$ of the sampled microbiomes, their abundances are expected to be inversely

261 correlated. An increase in this ratio is observed in phase B of the Soylent diet arm

262 compared to the regular diet arm, followed by a rapid return to baseline levels in phase

263 C. Previous studies have demonstrated associations between the relative abundance of

264 the phyla Bacteroidetes and Firmicutes and specific health outcomes. In particular, it

265 has been shown that a low Bacteroidetes to Firmicutes ratio is associated with obesity

266 in mice and humans $[20,21]$. Additionally, a low ratio is associated with ulcerative colitis

267 and Crohn's disease, which are types of inflammatory bowel disease [22]. Although

268 these associations have been strongly demonstrated, causality has not yet been

269 established in either case.

270 Although the participant pool is relatively small, similar sample sizes have articulated

271 clear results in other diet-related gut microbiome studies [6]. Nevertheless, missing data

272 for some samples presents a clear limitation to the study. We were unable to

273 troubleshoot these samples because sequencing was outsourced to uBiome Inc.

274 Additionally, the number of reads per sample was highly variable. Therefore, we had to

275 rarefy our samples to perform meaningful statistical analyses. Although we discarded

276 many reads from high-depth samples, rarefying has been shown to effectively mitigate

277 biases associated with sequencing depth variation [18]. 
278 Although the limitations of 16 S surveys are well understood (particularly the lack of

279 access to functional information), the accuracy of metagenome reconstruction is still

280 under debate and conclusions drawn from this method should be met with skepticism.

281 To confidently address these limitations, further studies involving transcriptomics and

282 metagenomics must be conducted to more accurately identify changes in gene

283 expression, gene function, and abundance resulting from a Soylent 2.0 dietary

284 intervention.

285 We conclude that a short-term interventional Soylent diet does not negatively impact the

286 composition of gut flora communities. As additional studies demonstrate the effect that

287 specific microbial consortia have on health, both food product manufacturers and

288 consumers should consider the gut microbiome as an essential component of human

289 health.

\section{Data Availability}

291 Raw sequencing reads in FASTQ format from this study were uploaded to EBl's

292 European Nucleotide Archive under the accession code PRJEB21752

293 (http://www.ebi.ac.uk/ena/data/view/PRJEB21752). 


\section{Acknowledgements}

295 We greatly appreciate the 84 backers of our Experiment crowdfunding campaign for 296 making this study possible. We thank Gwyneth Terry for assisting with the 297 administrative coordination of the project, as well as Shyam Bhakta and Michael 298 Shapira for helpful discussions. We also thank uBiome Inc. for their partnership in 299 performing sample processing and DNA sequencing.

\section{References}

301 1. Turnbaugh PJ, Ley RE, Mahowald MA, Magrini V, Mardis ER, Gordon JI. An

302 obesity-associated gut microbiome with increased capacity for energy harvest.

$303 \quad$ Nature. 2006;444: 1027-1031.

304 2. Halfvarson J, Brislawn CJ, Lamendella R, Vázquez-Baeza Y, Walters WA, Bramer

305 LM, et al. Dynamics of the human gut microbiome in inflammatory bowel disease.

$306 \quad$ Nat Microbiol. 2017;2: 17004.

307 3. Brandt LJ, Aroniadis OC, Mellow M, Kanatzar A, Kelly C, Park T, et al. Long-term

308 follow-up of colonoscopic fecal microbiota transplant for recurrent Clostridium

309 difficile infection. Am J Gastroenterol. 2012;107: 1079-1087.

310 4. Zhernakova A, Kurilshikov A, Bonder MJ, Tigchelaar EF, Schirmer M, Vatanen T, et

311 al. Population-based metagenomics analysis reveals markers for gut microbiome 
composition and diversity. Science. 2016;352: 565-569.

313 5. Smits SA, Marcobal A, Higginbottom S, Sonnenburg JL, Kashyap PC.

314 Individualized Responses of Gut Microbiota to Dietary Intervention Modeled in

$315 \quad$ Humanized Mice. mSystems. 2016;1. doi:10.1128/mSystems.00098-16

316 6. David LA, Maurice CF, Carmody RN, Gootenberg DB, Button JE, Wolfe BE, et al.

317 Diet rapidly and reproducibly alters the human gut microbiome. Nature. 2014;505:

$318 \quad 559-563$.

319 7. Rosa Foods I. Soylent — Food, intelligently designed [Internet]. [cited 4 Jun 2017].

320 Available: http://soylent.com

321 8. Almonacid DE, Kraal L, Ossandon FJ, Budovskaya YV, Cardenas JP, Bik EM, et al.

$32216 \mathrm{~S}$ rRNA gene sequencing and healthy reference ranges for 28 clinically relevant

323 microbial taxa from the human gut microbiome. PLoS One. 2017;12: e0176555.

324 9. ryanusahk/mycrobes_soylent_microbiome: Mycrobes Soylent Microbiome DNA

$325 \quad$ Analysis Pipeline. 2017; doi:10.5281/zenodo.822861

326 10. Edgar RC. Search and clustering orders of magnitude faster than BLAST.

327 Bioinformatics. 2010;26: 2460-2461.

328 11. Edgar RC, Haas BJ, Clemente JC, Quince C, Knight R. UCHIME improves sensitivity and speed of chimera detection. Bioinformatics. 2011;27: 2194-2200.

330 12. Rognes T, Flouri T, Nichols B, Quince C, Mahé F. VSEARCH: a versatile open 331 source tool for metagenomics. PeerJ. 2016;4: e2584. 
332 13. Schloss PD, Westcott SL, Ryabin T, Hall JR, Hartmann M, Hollister EB, et al.

333 Introducing mothur: open-source, platform-independent, community-supported

334 software for describing and comparing microbial communities. Appl Environ

$335 \quad$ Microbiol. 2009;75: 7537-7541.

14. McDonald D, Price MN, Goodrich J, Nawrocki EP, DeSantis TZ, Probst A, et al. An improved Greengenes taxonomy with explicit ranks for ecological and evolutionary

15. Caporaso JG, Bittinger K, Bushman FD, DeSantis TZ, Andersen GL, Knight R. analyses of bacteria and archaea. ISME J. 2012;6: 610-618. PyNAST: a flexible tool for aligning sequences to a template alignment.

16. Price MN, Dehal PS, Arkin AP. FastTree 2--approximately maximum-likelihood Bioinformatics. 2010;26: 266-267.

344 17. Caporaso JG, Kuczynski J, Stombaugh J, Bittinger K, Bushman FD, Costello EK, et trees for large alignments. PLoS One. 2010;5: e9490.

18. Weiss S, Xu ZZ, Peddada S, Amir A, Bittinger K, Gonzalez A, et al. Normalization and microbial differential abundance strategies depend upon data characteristics. Microbiome. BioMed Central; 2017;5: 27. and Nosocomial Diarrhea. J Clin Microbiol. 2013;51: 2884-2892. 
353 20. Ley RE, Bäckhed F, Turnbaugh P, Lozupone CA, Knight RD, Gordon Jl. Obesity

354 alters gut microbial ecology. Proc Natl Acad Sci U S A. 2005;102: 11070-11075.

355 21. Ley RE, Turnbaugh PJ, Klein S, Gordon JI. Microbial ecology: human gut microbes

356 associated with obesity. Nature. 2006;444: 1022-1023.

357 22. Zhou Y, Zhi F. Lower Level of Bacteroides in the Gut Microbiota Is Associated with

358 Inflammatory Bowel Disease: A Meta-Analysis. Biomed Res Int. 2016;2016:

$359 \quad 5828959$. 
${ }_{361}$ Supporting Information

362 S1 Fig. Nutrition Facts for the Soylent 2.0 meal replacement drink. Participants in

363 the Soylent diet group consumed 5 servings of Soylent per day during phase B of the 364 study.

365

366 S2 Fig. Sequencing depth was variable among samples. The 1st, 2nd, and 3rd 367 quartiles of sequencing depths were 8335 , 83777, and 208145 reads, respectively.

S3 Fig. a-diversity remains consistent throughout a Soylent dietary intervention.

370 The Soylent dietary intervention did not result in a significant change in a-diversity

371 (Soylent A to Soylent B), as shown by the Shannon-Wiener $(p=0.279)$ and Gini-Simpson

$372(p=0.999)$ metrics. Standard error is shown.

373

374 S4 Fig. Principle Coordinate Analysis visualization of two Unifrac variants.

375 Unweighted UniFrac, which considers the binary presence or absence of each OTU,

376 reveals that samples cluster strongly by participant (indicated by color) and not by diet

377 (denoted by 's or x's). Similar but less apparent trends are seen with the Weighted 378 UniFrac metric, which weights OTUs based on fractional abundance as well.

380 S1 Table. The table links participant IDs to diet groups and collected stool samples.

381 Samples that returned no reads or were discarded due to low sequencing depth are 382 denoted. 
bioRxiv preprint doi: https://doi.org/10.1101/200881; this version posted October 13,2017 . The copyright holder for this preprint (which was not certified by peer review) is the author/funder, who has granted bioRxiv a license to display the preprint in perpetuity. It is made available under aCC-BY 4.0 International license.

\section{S1 File. Daily Electronic Log Form}

384

385 S2 File. Validation and discussion of rarifying 


\section{Enrollment}

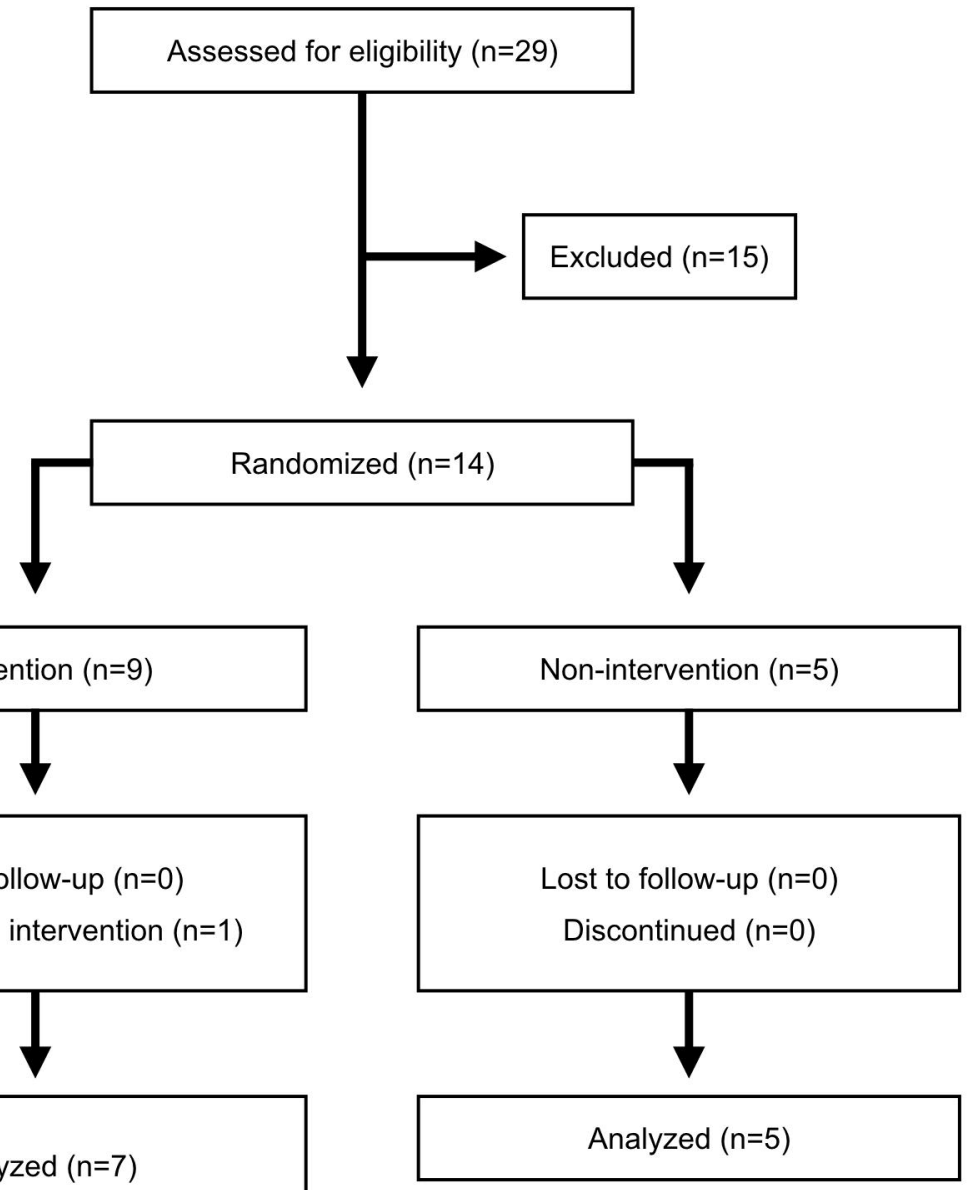

\section{Analysis}

$$
\text { Analyzed ( } n=7)
$$

Excluded from analysis

No sequencing data $(n=1)$ 


\begin{tabular}{|c|c|c|c|c|c|c|c|c|c|c|}
\hline \multirow[b]{2}{*}{ Day } & \multicolumn{2}{|c|}{ Phase A } & \multicolumn{4}{|c|}{ Phase B } & \multicolumn{4}{|c|}{ Phase C } \\
\hline & 1 & 2 & 3 & 4 & 5 & 6 & 7 & 8 & 9 & 10 \\
\hline Microbiome Sampling & & & & & & & & & & \\
\hline Soylent Group ( $n=9)$ & \multicolumn{2}{|c|}{ regular diet } & \multicolumn{4}{|c|}{ Soylent diet } & \multicolumn{4}{|c|}{ regular diet } \\
\hline Control Group $(n=5)$ & \multicolumn{2}{|c|}{ regular diet } & \multicolumn{4}{|c|}{ regular diet } & \multicolumn{4}{|c|}{ regular diet } \\
\hline
\end{tabular}




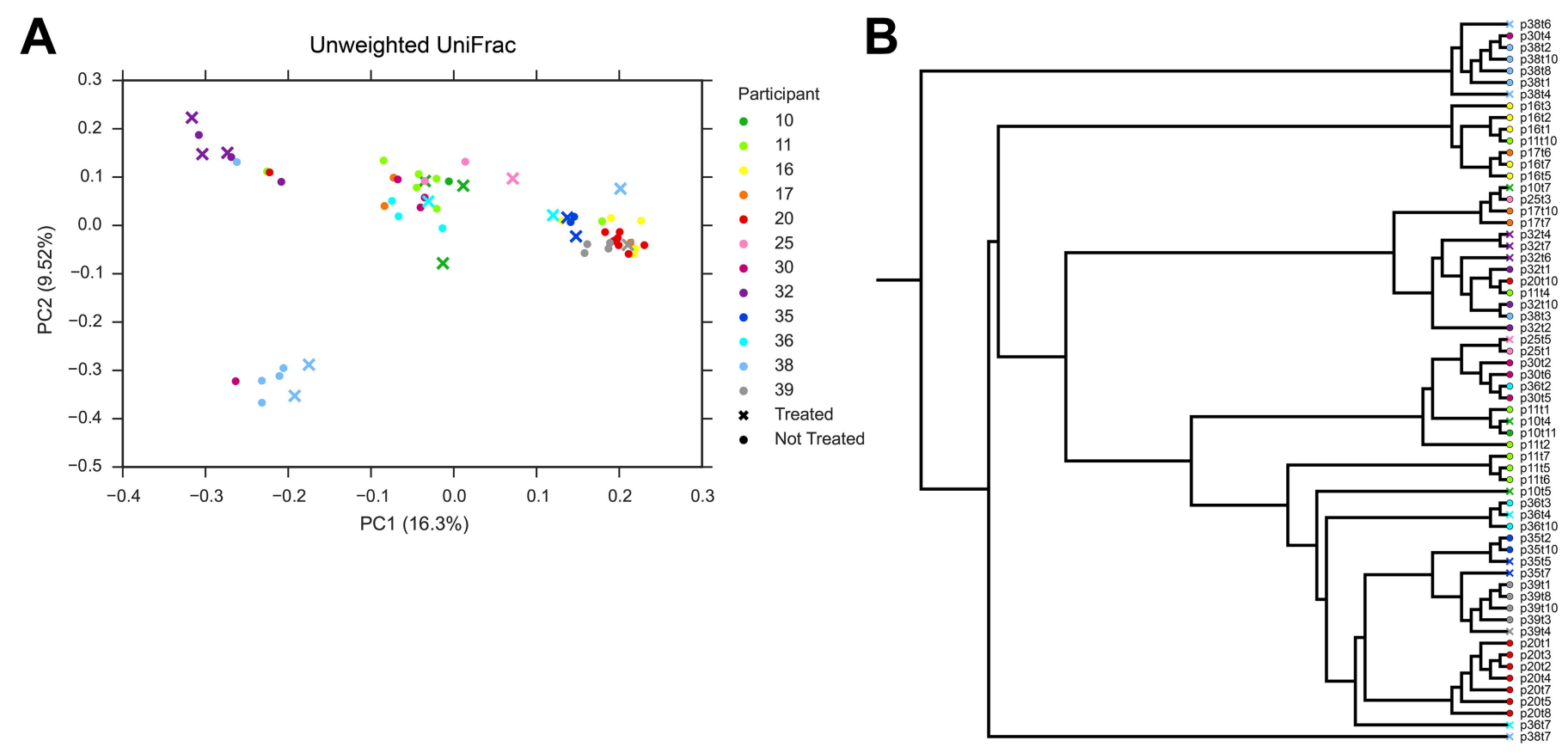



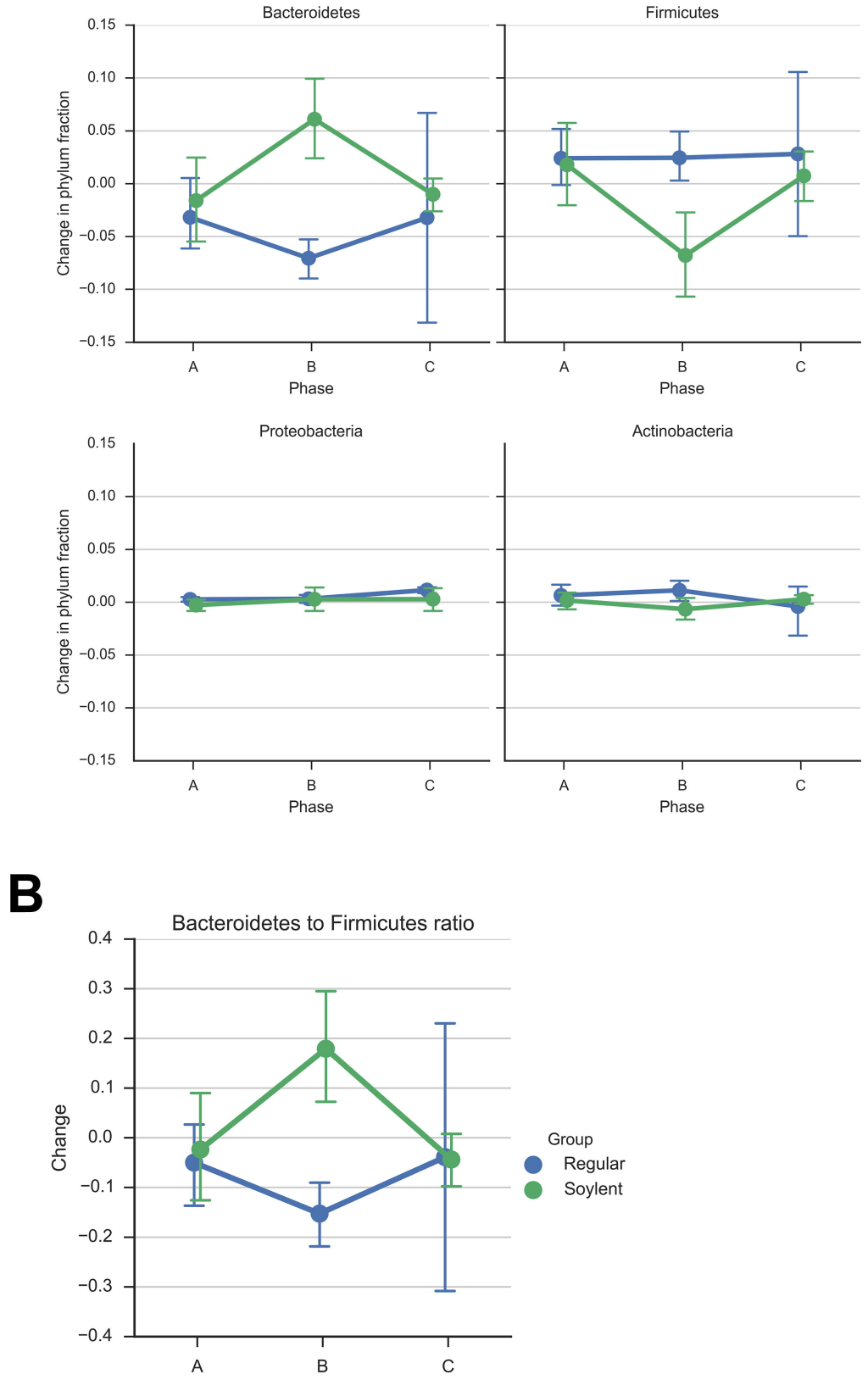